\title{
Dual upconversion nanophotoswitch for security encoding
}

\author{
Weijing $\mathrm{Yao}^{1 \dagger}$, Qingyong Tian ${ }^{1 \dagger}$, Bin Tian ${ }^{1}$, Mengxiao $\mathrm{Li}^{1}$, Huanjun Wang ${ }^{1}$, Pan Zeng ${ }^{1}$, Li Liu ${ }^{1}$, \\ He Zheng ${ }^{2}$ and $\mathrm{Wei} \mathrm{Wu}^{1,3,4^{*}}$
}

\begin{abstract}
Light-harvesting lanthanide ions $\left(\mathrm{Ln}^{3+}\right)$ doped $\mathrm{NaYF}_{4}$ inks could provide polychromatic patterns for opposing counterfeiting commodity infestation because of their distinctive upconversion photoluminescence (UPL) properties. Herein, three kinds of core-triple-shell $\mathrm{Ln}^{3+}$ ions doped $\mathrm{NaYF}_{4}$ upconversion nanocrystals (UCNCs) are synthesized through modified high-temperature coprecipitation, which demonstrate excellent UPL properties of independent emitting colors under 808 or $980 \mathrm{~nm}$ laser excitation. Additive mixing three kinds of $808 \mathrm{~nm}$ emitted red-green-blue (RGB) UCNCs colloid solution can precisely regulate the emissions of the suspension for achieving full-color display. The as-obtained RGB three-primary colors induced by $808 \mathrm{~nm}$ laser accomplish broader color gamut than traditional standard RGB (sRGB) model and printing cyan-magenta-yellow (CMY) model. In addition, various China zodiac patterns and complex multicolor images are printed by the as-formulated UCNCs inks through screen printing technology. The printed patterns present colorful and polychromatic sequential toning visualization patterns under $808 \mathrm{~nm}$ excitation, while present another succession of gradually changed versatile patterns under $980 \mathrm{~nm}$ excitation. As a proof of concept, transparent polyvinyl chloride (PVC) self-adhesive anti-counterfeiting label is attached to the bottle of wine package for practical application. The demonstration of multiple model patterns of Chinese zodiac and poetry images based on these core-tripleshell UCNCs can be selected as a conceivable substitute of traditional single model patterns, underlining the full-color anti-counterfeiting level.
\end{abstract}

Keywords: Upconversion, core-triple-shell UCNCs, wide color gamut, full-color model, anti-counterfeiting

\section{INTRODUCTION}

With the continuous development of high-technology and commodity market, the interpenetration of various anti-counterfeiting models is demanded to set up layers of obstacles and resistance for counterfeits [1-4]. Encoding high-density and complicated data into an independent unit has been proved its significance to improve information security level [5-7]. The emission color of fluorescent materials are especially suitable for encoding information and optical multiplexing [8-11]. For instance, traditional semiconductor quantum dots (based on down-conversion (DC) mechanism, convert short wavelength to long wavelength) and fluorescent dyes could be readily printed as labels to form colorful code under certain wavelength irradiation [12-14]. However, those materials suffer from the disadvantages of high poisonousness, easy detection and relative unstability [15-17]. Alternatively, $\mathrm{Ln}^{3+}$ ions doped upconversion (UC) materials are particularly suitable for security application because of intrinsic upconversion photoluminescence (UPL) properties, including anti-Stokes fluorescence (converted long wavelength to short wavelength) $[16,18]$, tunable multicolor emissions under nearinfrared (NIR) light excitation $[19,20]$, perfect biological compatibility and long fluorescent lifetime [21,22].

In the past few years, extensive studies have been devoted to investigating multi-color tuning of lanthanide doping materials, where the nanocrystals are synthsized through high-temperature coprecipitation method and solvothermal process [23-26]. The feature of UC materials is that the eventual emitting color could only be

\footnotetext{
${ }^{1}$ Laboratory of Printable Functional Nanomaterials and Printed Electronics, School of Printing and Packaging, Wuhan University, Wuhan 430072, China

${ }^{2}$ School of Physics and Technology, Wuhan University, Wuhan 430072, China

${ }^{3}$ Shenzhen Research Institute of Wuhan University, Shenzhen 518057, China

${ }^{4}$ National \& Local Joint Engineering Research Center of Advanced Packaging Materials Developing Technology, Hunan University of Technology, Zhuzhou 412007, China

These authors contributed equally to this work.

* Corresponding author (email: weiwu@whu.edu.cn)
} 
regulated under single model wavelength irradiation. However, the patterns based on single model fluorescence can be easily reproduced and are inadequate to conceal the authentic infromation. And in order to achieve optimal color displaying, continuous optimization of experimental parameters and dopant concentrations is required. One potential tactic used to simplify encoding process is to combine multiple model emissions with nanocrystals for replacing single model emissions [2729]. Incorporating DC and UC mechanisms into an system for acheiving multiple model fluorescence has been implemented [27,30,31]. For instance, in our previous work, carbon quantum dots (CDs) were combined with $\mathrm{UC} \mathrm{NaYF}$ : $: \mathrm{Er}, \mathrm{Yb}(\mathrm{Tm})$ to realize dual-mode fluorescent patterns under 365 and $980 \mathrm{~nm}$ excitation, indicating outstanding anti-counterfeiting application [30]. Although they can serve for security, the drawbacks of presenting colors with relative narrow color gamut limit their further utilization. Various techniques coupled with $\mathrm{Ln}^{3+} \mathrm{UC}$ materials have been developed for security application, including radio frequency identification (RFID) tags [32], inkjet printing [7] and laser holograms [33,34]. However, these techniques depend on high-cost production equipment, clean-room facilities and complex preparation procedure. Therefore, how to acheive true fullcolor and tunable fluorescence patterns through a simple technique becomes an urgent challenge in the anticounterfeiting fields.

To address the above challenges, three types of coremultipe-shell UC nanocrystals (UCNCs) coupled with low-cost screen printing technique have been used as a promising strategy in our study. In the various layered UC nanostructures, diverse sensitizers and activators were confined in different layers for displaying multicolor. Intriguingly, the core-triple-shell UC nanoparticles display pure RGB color under $808 \mathrm{~nm}$ excitation and accomplish broad array of colors through straightforward additive mixing different volume ratio of RGB UCNCs colloidal solution. After inks formulation process, we employed the as-obtained UCNCs-poly(acrylic acid) (PAA) inks to screen-print designable and versatile patterns and complex large-area images, achieving the fullcolor model anti-counterfeiting for wine package.

\section{EXPERIMENTAL SECTION}

\section{Materials and reagents}

$\mathrm{YCl}_{3} \cdot 6 \mathrm{H}_{2} \mathrm{O}$ (99.99\% metals basis), $\mathrm{YbCl}_{3} \cdot 6 \mathrm{H}_{2} \mathrm{O}(99.9 \%$ metals basis), $\mathrm{NdCl}_{3} \cdot 6 \mathrm{H}_{2} \mathrm{O}$ (99.9\% metals basis), $\mathrm{TmCl}_{3}$. $6 \mathrm{H}_{2} \mathrm{O}$ (99.99\% metals basis), $\mathrm{ErCl}_{3} \cdot 6 \mathrm{H}_{2} \mathrm{O}(99.995 \%$ metals basis), 1-octadecene $\left(\mathrm{C}_{18} \mathrm{H}_{36}, \mathrm{ODE}\right)$, oleic acid (OA, 85\%) and poly (acrylic acid) $50 \%$ solution (PAA, $M_{\mathrm{w}}=3000$ ) were purchased from Aladdin Chemistry Co., Ltd. $\mathrm{NaOH}$ (AR), $\mathrm{NH}_{4} \mathrm{~F}(\mathrm{AR})$, methanol $\left(\mathrm{CH}_{4} \mathrm{O}, \mathrm{AR}\right)$, cyclohexane $\left(\mathrm{C}_{6} \mathrm{H}_{12}\right)$ and ethanol were obtained from Sinopharm Chemical Reagent Co., Ltd.

\section{Preparation of $\mathrm{NaYF}_{4}$ core-triple-shell structured nanocrystals}

The $\mathrm{NaYF}_{4}$ core-triple-shell nanocrystals were fabricated by a modified high-temperature coprecipitation process. Typically, the step-by-step synthesis of $\mathrm{NaYF}_{4}: \mathrm{Yb}^{3+}$, $\mathrm{Tm}^{3+} @ \mathrm{NaYF}_{4}: \mathrm{Nd}^{3+} @ \mathrm{NaYF}_{4} @ \mathrm{NaYF}_{4}: \mathrm{Yb}^{3+}, \mathrm{Er}^{3+}$ nanocrystals (denoted as TNYE UCNCs, P1) was illustrated as follows:

Synthesis of $\mathrm{NaYF}_{4}: \mathrm{Yb}^{3+}, \mathrm{Tm}^{3+}$ core structured nanocrystals (denoted as T UCNCs). For the preparation of $\mathrm{NaYF}_{4}: \mathrm{Yb}^{3+}, \mathrm{Tm}^{3+}$ nanoparticles, $1 \mathrm{mmol}$ of $\mathrm{ReCl}_{3}$ precursor (Y:Yb:Tm $=80 \%: 18 \%: 2 \%$ ) was mixed into a $50 \mathrm{~mL}$ three-necked flask with adding $7.5 \mathrm{~mL}$ of $\mathrm{OA}$ and $15 \mathrm{~mL}$ of ODE. The suspension was heated to $160^{\circ} \mathrm{C}$ under argon atmosphere and kept at this temperature for $30 \mathrm{~min}$ while stirring. When it was cooled down to $35^{\circ} \mathrm{C}$ naturally, a mixture of $\mathrm{NaOH}\left(1 \mathrm{~mol} \mathrm{~L}^{-1}, 2.5 \mathrm{~mL}\right)$ and $\mathrm{NH}_{4} \mathrm{~F}$ methanol solution $\left(0.4 \mathrm{~mol} \mathrm{~L}^{-1}, 10 \mathrm{~mL}\right)$ were immediately injected into the flask. And then the temperature of resultant mixture was increased to $50^{\circ} \mathrm{C}$ and maintained for $30 \mathrm{~min}$ to ensure the complete nucleation. Afterward, the reaction mixture was heated to $110^{\circ} \mathrm{C}$ for removing methanol and raised up to $300^{\circ} \mathrm{C}$ for $1.5 \mathrm{~h}$ immediately. Eventually, the resultant mixture was cooled down to $30^{\circ} \mathrm{C}$ gently under the protecting of slow argon flow. The precipitates were centrifuged and washed with ethanol, cyclohexane and water for several times successively and dispersed in cyclohexane for further utilization.

Synthesis of $\mathrm{NaYF}_{4}: \mathrm{Yb}^{3+}, \mathrm{Tm}^{3+} @ \mathrm{NaYF}_{4}: \mathrm{Nd}^{3+}$ coreshell structured nanocrystals (denoted as TN UCNCs). The overcoating of $\mathrm{NaYF}_{4}: \mathrm{Nd}^{3+}$ shell layer was carried out with the similar process as above-mentioned preparation process of T UCNCs except that $2 \mathrm{mmol}$ of $\mathrm{ReCl}_{3}$ precursor ( $\mathrm{Y}: \mathrm{Nd}=80 \%: 20 \%$ ) was introduced. And the $\mathrm{T}$ UCNCs cyclohexane colloidal solution $\left(0.25 \mathrm{~mol} \mathrm{~L}^{-1}\right.$, $4 \mathrm{~mL}$ ) was rapidly pipetted into three-necked flask which was stirred for 5 min before adding the mixture of $\mathrm{NaOH}$ and $\mathrm{NH}_{4} \mathrm{~F}$ methanol solution.

Synthesis of $\mathrm{NaYF}_{4}: \mathrm{Yb}^{3+}, \mathrm{Tm}^{3+} @ \mathrm{NaYF}_{4}: \mathrm{Nd}^{3+} @ \mathrm{NaYF}_{4}$ core-double-shell structured nanocrystals (denoted as TNY UCNCs). The overcoating of the second $\mathrm{NaYF}_{4}$ shell layer was carried out as similar as the above-mentioned preparation process of TN UCNCs but $1 \mathrm{mmol}$ of 
$\mathrm{YCl}_{3}$ precursor was introduced. Then, $4 \mathrm{~mL}$ of $0.25 \mathrm{~mol} \mathrm{~L}^{-1}$ TN UCNCs cyclohexane colloidal solution was rapidly pipetted into three-necked flask which was stirred for 5 min before injecting the mixture of $\mathrm{NaOH}$ and $\mathrm{NH}_{4} \mathrm{~F}$ methanol solution.

Synthesis of $\mathrm{NaYF}_{4}: \mathrm{Yb}^{3+}, \mathrm{Tm}^{3+} @ \mathrm{NaYF}_{4}: \mathrm{Nd}^{3+} @ \mathrm{NaYF}_{4} @$ $\mathrm{NaYF}_{4}: \mathrm{Yb}^{3+}, \mathrm{Er}^{3+}$ core-triple-shell structured nanocrystals (denoted as TNYE UCNCs). The overcoating of the third shell layer, $\mathrm{NaYF}_{4}: \mathrm{Yb}^{3+}, \mathrm{Er}^{3+}$, was carried out as similar as the above-mentioned preparation of TNY UCNCs except that $1 \mathrm{mmol}$ of $\mathrm{ReCl}_{3}$ precursor (Y:Yb:Er= 80\%:18\%:2\%) was introduced before injecting the mixture of $\mathrm{NaOH}$ and $\mathrm{NH}_{4} \mathrm{~F}$ methanol solution. The TNY UCNCs cyclohexane colloidal solution $\left(0.25 \mathrm{~mol} \mathrm{~L}^{-1}\right.$, $4 \mathrm{~mL}$ ) was rapidly pipetted into the three-necked flask while stirring for $5 \mathrm{~min}$. The resulting products were collected and washed with ethanol, cyclohexane and water for five times successively, and then dispersed in cyclohexane for further application.

Moreover, the synthesis process of $\mathrm{NaYF}_{4}: \mathrm{Yb}^{3+}, \mathrm{Tm}^{3+} @$ $\mathrm{NaYF}_{4} @ \mathrm{NaYF}_{4}: \mathrm{Nd}^{3+} @ \mathrm{NaYF}_{4}: \mathrm{Yb}^{3+}, \mathrm{Er}^{3+}$ nanocrystals (denoted as TYNE UCNCs, P2) and $\mathrm{NaYF}_{4}: \mathrm{Er}^{3+}, \mathrm{Tm}^{3+} @$ $\mathrm{NaYF}_{4}: \mathrm{Nd}^{3+} @ \mathrm{NaYF}_{4} @ \mathrm{NaYF}_{4}: \mathrm{Yb}^{3+}, \mathrm{Er}^{3+}$ nanocrystals (denoted as ETNYE UCNCs, P3) were similar with the preparation of TNYE UCNCs. While, the precursors of $\mathrm{ReCl}_{3}$ were replaced as follows: $\mathrm{P} 2, \mathrm{Y}: \mathrm{Yb}: \mathrm{Tm}=80 \%: 18 \%$ : $2 \% @ \mathrm{Y}=100 \% @ \mathrm{Y}: \mathrm{Nd}=80 \%: 20 \% @ \mathrm{Y}: \mathrm{Yb}: \mathrm{Er}=80 \%: 18 \%$ : 2\%; P3, Y:Er:Tm = 88\%:10\%:2\%@Y:Nd = 80\%:20\%@Y = 100\%@Y:Yb:Er = 80\%:18\%:2\%. All the preparation parameters of samples are listed in Table S1 (Supplementary information).

\section{Fabrication of anti-counterfeiting patterns by screen printing technology}

Two screen plates with designable and versatile patterns were obtained for next utilization, which possess 300 mesh counts. The as-prepared UCNCs $(90 \mathrm{mg})$ were mixed with PAA ( $3 \mathrm{~mL})$ to formulate the UCNCs fluorescent inks, followed by screen printing versatile patterns on white cardboard.

\section{Characterizations}

Transmission electron microscopy (TEM), high-resolution transmission electron microscopy (HRTEM) of samples were carried out using a JEM-2100 TEM with $200 \mathrm{kV}$ accelerating voltage. Scanning electron microscopy (SEM) was recorded by a cold-field emission $\mathrm{Hi}$ tachi S-4800 SEM. The room-temperature fluorescent spectra were characterized by using a Hitachi F-4600 spectrophotometer equipped with external $808 \mathrm{~nm}$
(Changchun New Industries Optoelectronics Tech Co., Ltd) and $980 \mathrm{~nm}$ (Changchun Bright Optoelectronics Co., Ltd) fiber coupled laser system. The information and signatures of the patterns were recorded by using a Nikon D750 camera equipped with high throughout filters (400-700 nm) under adjustable excited source of $980 \mathrm{~nm}$ laser or $808 \mathrm{~nm}$ laser. The laser power density of $980 \mathrm{~nm}$ laser and $808 \mathrm{~nm}$ laser for the photoluminescence (PL) measurements were ca. 48 and $80 \mathrm{~W} \mathrm{~cm}^{-2}$, respectively.

\section{RESULTS AND DISCUSSION}

For rational design of color-tunable UC nanoparticles, hexagonal-phase $\mathrm{NaYF}_{4}$ is selected as the matrix material. And four types of $\mathrm{Ln}^{3+}\left(\mathrm{Yb}^{3+}, \mathrm{Nd}^{3+}, \mathrm{Tm}^{3+}, \mathrm{Er}^{3+}\right)$ are incorporated into specific layer with certain molar concentrations. The typical layer-by-layer epitaxial growth route is used to synthesize the TNYE UCNCs, which is schematic displayed in Fig. 1a. TEM and corresponding HRTEM images are illustrated in Fig. $1 b-i$, respectively. TEM images in Fig. 1b-f present the highly uniform and gradually increased core-multishell nanocrystals along with the synthesis procedures. Comparatively, the morphologies evolve from spheroidicity to short rod shape and the average lengths of UCNCs increase from $\sim 34$ to $\sim 68 \mathrm{~nm}$. The size distrubution statistics are inseted in Fig. 1b-e. Meanwhile, corresponding HRTEM of the core-triple-shell UCNCs (Fig. 1f-i) present well-defined lattice fringes of 0.517 and $0.298 \mathrm{~nm}$, indexing to the (100) and (110) plane of $\beta-\mathrm{NaYF}_{4}$, respectively. They are verified by the results of fast Fourier transform (FFT, insets of Fig. 1g-i). Clearly, the lattice orientation has been changed after coating with the shell layer. Additionally, the SEM images and size distributions of other two kinds of TYNE UCNCs and ETNYE UCNCs are shown in Figs S1 and S2 (Supplementary information), respectively. The sequence evolution of the morphologies proves the successful construction of core-triple-shell UCNCs.

The crystallographic phases of T UCNCs, TN UCNCs, TNY UCNCs, TNYE UCNCs are confirmed by XRD patterns, as shown in Fig. 2. The XRD pattern of $T$ UCNCs demonstrates that all positions and relative intensity are consistent with the standard JCPDS card (281192) of $\beta-\mathrm{NaYF}_{4}$. No external diffraction peaks appear with layer-by-layer epitaxial growing of different shells, because the doping of $\mathrm{Ln}^{3+}$ does not change the crystal structure of $\mathrm{NaYF}_{4}$ mixture. Absence of any noticeable impurity phase indicates that the core-triple-shell UCNCs are successfully synthesized.

The optical properties of $\mathrm{NaYF}_{4}$ nanoparticles can be 


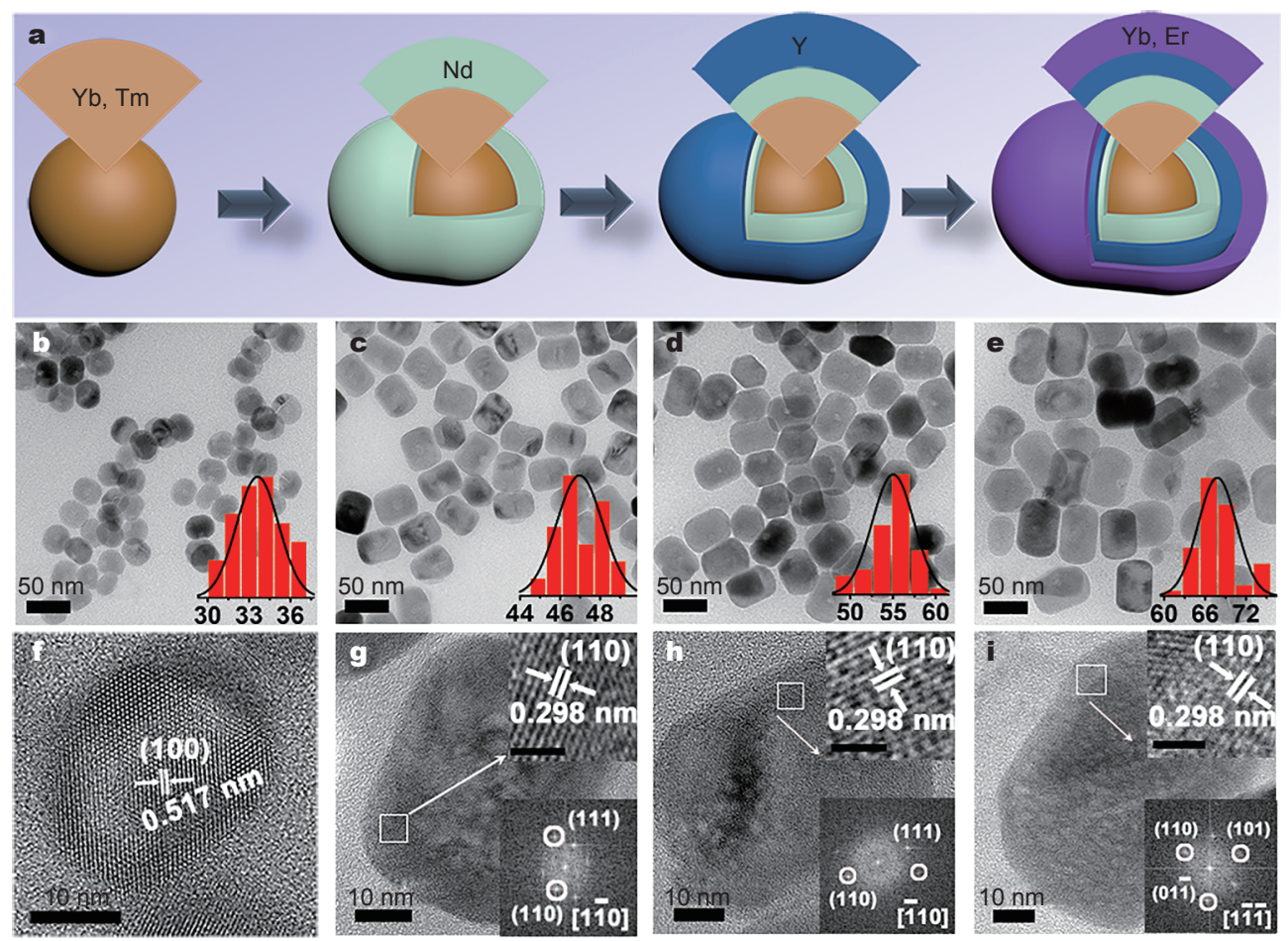

Figure 1 Typical schematic illustration of the synthesis procedure of TNYE UCNCs (a), TEM images (b-e, the insets are corresponding size distributions), corresponding HRTEM (f-i, the insets are corresponding FFT patterns) of T UCNCs, TN UCNCs, TNY UCNCs and TNYE UCNCs, respectively.

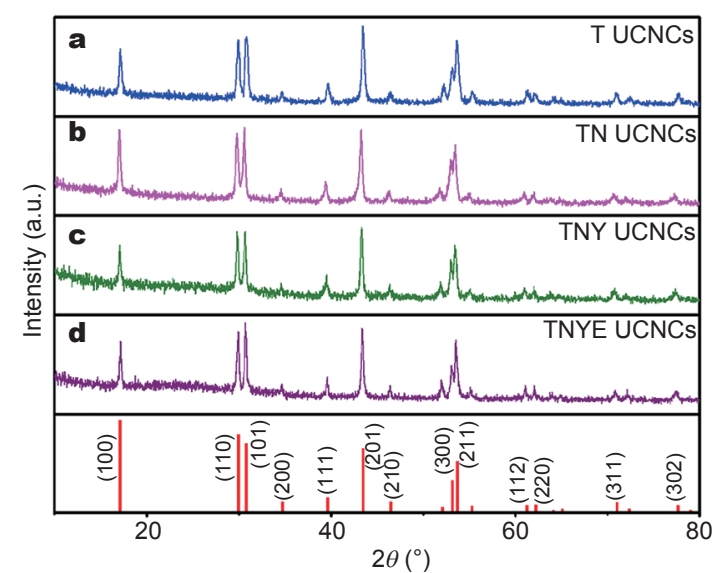

Figure 2 XRD patterns of T UCNCs (a), TN UCNCs (b), TNY UCNCs (c), TNYE UCNCs (d) and the standard JCPDS card of $\beta-\mathrm{NaYF}_{4}(28-$ 1192).

tailored by adjusting the $\mathrm{Ln}^{3+}$ dopants in both core and shell layers, which is an efficient method to incorporate different UC emissions into one single nanocrystal $[35,36]$. Therefore, the rationally designed $\mathrm{NaYF}_{4}$ based core-triple-shell nanoparticles are expected to emit tun- able color under irradiation of NIR lasers. Subsequently, the UPL properties of core-triple-shell nanoparticles are investigated, as shown in Fig. 3. Under $808 \mathrm{~nm}$ irradiation, the TNYE UCNCs present overwhelming blue color emission peak centered at $477 \mathrm{~nm}$ (Fig. 3a). Interestingly, there are also green emission peaks at 523 and $543 \mathrm{~nm}$ with lower intensity accompanying with the blue emission when excited under $980 \mathrm{~nm}$ laser (Fig. 3a). Eventually, the TNYE UCNCs in cyclohexane collodial solution exhibit blue (B) and cycan color under irradiation of 808 and $980 \mathrm{~nm}$ lasers with filtering out of UV/IR interference signal, respectively (inset of Fig. 3a). Meanwhile, the UPL spectra of TYNE UCNCs are shown in Fig. 3b, which gave rise to two divergent green peaks at 523 and $543 \mathrm{~nm}$ under excitation at $808 \mathrm{~nm}$ laser, and a dominant blue peak under excitation at $980 \mathrm{~nm}$ laser. Thus, the photograph of corresponding colloidal solution appears pure green $(\mathrm{G})$ and blue color (B) under 808 and $980 \mathrm{~nm}$ irradiation (inset of Fig. 3b). Additionally, Fig. 3c presents the fluorescence spectra of ETNYE UCNCs, which yield a sharp red peak $(657 \mathrm{~nm})$ under $808 \mathrm{~nm}$ laser irradiation, and two peaks (543 and $657 \mathrm{~nm}$ ) under $980 \mathrm{~nm}$ laser irradiation. The ETNYE UCNCs in cyclo- 

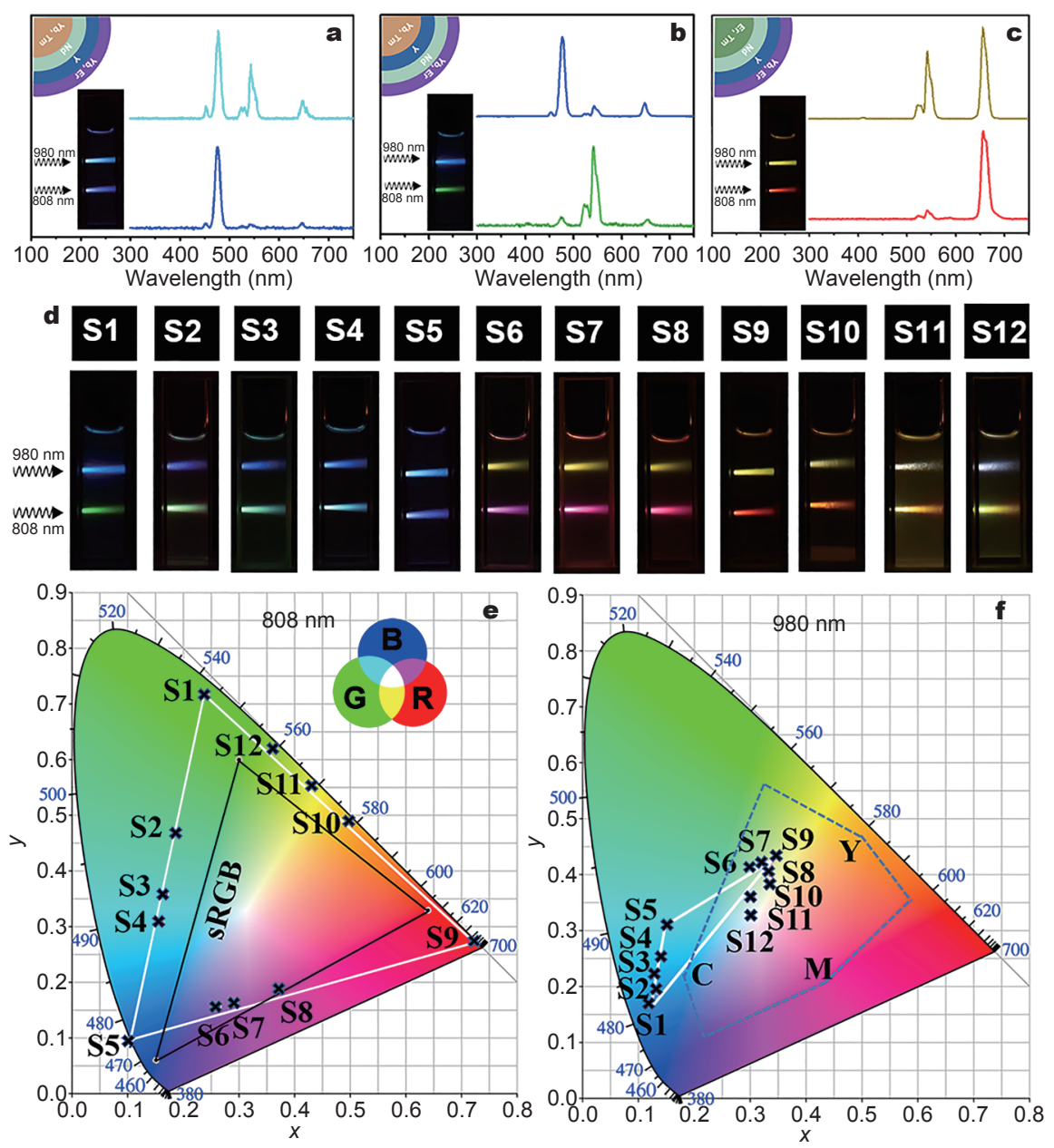

Figure 3 Tunable UC emission spectra of (a) TNYE UCNCs, (b) TYNE UCNCs and (c) ETNYE UCNCs. Top inset: corresponding schematic coretriple-shell structures. Left inset: corresponding multi-color luminescence images of UCNCs in cyclohexane solution under the excitation of 808 and $980 \mathrm{~nm}$ lasers. Fluorescence photographs (d) of full-color tunable samples obtained by additive mixing certain amount of two kinds of as-prepared UCNCs cyclohexane solution. Color gamut of emissions under $808 \mathrm{~nm}$ (e) and $980 \mathrm{~nm}$ (f) marked on the CIE chromaticity diagram. The color spaces of sRGB and printing CMY are marked with white and blue triangles, respectively.

hexane solution exhibit the pure red $(\mathrm{R})$ and overlapped yellow color (Fig. 3c) under 808 and $980 \mathrm{~nm}$ irradiation, respectively. Additive mixing of ink is a straightforward approach to tune multi-color and extend the color gamut according to the overlay principle of RGB colors. Herein, we set the as-obtained RGB colors under $808 \mathrm{~nm}$ irradiation as the basic coordinates for tuning color display. To achieve this aim, certain amounts of two types of UCNCs in cyclohexane solution with similar fluorescence intensity under $808 \mathrm{~nm}$ excitation are mixed physically (denoted as P1, P2 and P3, respectively). The detail mixing parameters are listed in Table 1 . By adjusting the volume ratio of $\mathrm{P} 1$ and $\mathrm{P} 2$, or $\mathrm{P} 2$ and $\mathrm{P} 3$, or $\mathrm{P} 3$ and $\mathrm{P} 1$ from $3 / 0$ to $0 / 3$, respectively, the gradient color emission of UCNCs colloidal solution can be gradually generated (Fig. 3d). Furthermore, corresponding fluorescent spectra of UCNCs solution under different volume ratio are also explored and the detail coordinates are marked on the CIE chromaticity diagram (Table 1 and Figs S3-S5). Remarkably, they are accessible to broader color gamut (marked by the dark triangle, Fig. 3e) compared with sRGB (marked by the white triangle, Fig. 3e) and printing CMY (marked by blue dotted line of triangle, Fig. 3f) [37], providing essential condition for multi-color anticounterfeiting model.

Theoretically, varied emission engineering is derived from doping $\mathrm{Ln}^{3+}$ ions that can absorb the different wavelength excitation light. $\mathrm{Nd}^{3+}$ and $\mathrm{Yb}^{3+}$ as ideal sensiti- 
Table 1 Different additive mixing proportions of UCNCs colloids solution and corresponding CIE color coordinates

\begin{tabular}{|c|c|c|c|c|c|c|c|}
\hline \multirow{2}{*}{\multicolumn{4}{|c|}{ Proportion }} & \multicolumn{4}{|c|}{ Emission colors of CIE color coordinates } \\
\hline & & & & \multicolumn{2}{|c|}{$808 \mathrm{~nm}$} & \multicolumn{2}{|c|}{$980 \mathrm{~nm}$} \\
\hline Samples & P1 & $\mathrm{P} 2$ & P3 & $\mathrm{X}$ & $\mathrm{Y}$ & $\mathrm{X}$ & $\mathrm{Y}$ \\
\hline S1 & 0 & 3 & 0 & 0.2410 & 0.7176 & 0.1204 & 0.1685 \\
\hline S2 & 1 & 2 & 0 & 0.1867 & 0.4675 & 0.1350 & 0.1910 \\
\hline S3 & 1.5 & 1.5 & 0 & 0.1677 & 0.3581 & 0.1300 & 0.2200 \\
\hline S4 & 2 & 1 & 0 & 0.1597 & 0.3082 & 0.1423 & 0.2542 \\
\hline S5 & 3 & 0 & 0 & 0.1072 & 0.0993 & 0.1523 & 0.3107 \\
\hline S6 & 2 & 0 & 1 & 0.2621 & 0.1564 & 0.3266 & 0.4092 \\
\hline S7 & 1.5 & 0 & 1.5 & 0.2937 & 0.1629 & 0.3344 & 0.4217 \\
\hline S8 & 1 & 0 & 2 & 0.3721 & 0.1881 & 0.3374 & 0.3999 \\
\hline S9 & 0 & 0 & 3 & 0.7239 & 0.2761 & 0.3377 & 0.4245 \\
\hline S10 & 0 & 1 & 2 & 0.5000 & 0.4890 & 0.3355 & 0.3851 \\
\hline S11 & 0 & 1.5 & 1.5 & 0.4313 & 0.5578 & 0.3051 & 0.3573 \\
\hline S12 & 0 & 2 & 1 & 0.3636 & 0.6231 & 0.2992 & 0.3249 \\
\hline sR & 1 & I & I & 0.6400 & & 0.3000 & \\
\hline$s G$ & I & I & I & 0.3000 & & 0.6000 & \\
\hline $\mathrm{sB}$ & 1 & 1 & 1 & 0.1500 & & 0.0600 & \\
\hline
\end{tabular}

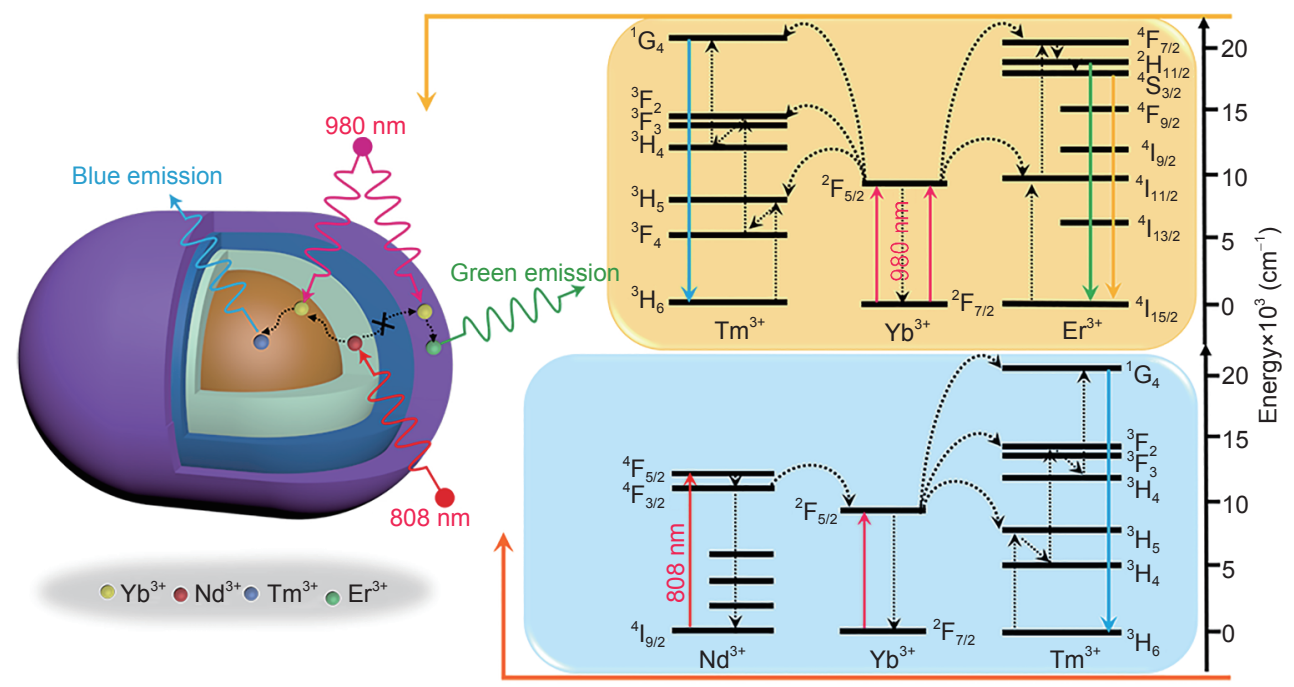

Figure 4 Schematic design of detail UC process in core-triple-shell TNYE UCNCs nanostructure managing complicated energy transfer.

zers possess large and efficient absorption cross section at 808 and $980 \mathrm{~nm}$, respectively [35,38,39]. Between the $\mathrm{Yb}^{3+}$ and $\mathrm{Nd}^{3+}$ ions doped layers, a $\mathrm{NaYF}_{4}$ layer is served as an energy-transfer barrier to prevent the bilateral energy transfer. The proposed UC processes of three types of UCNCs are depicted in Figs 4 and 5 [35]. In a typical UC mechanism of TNYE UCNCs, $\mathrm{Nd}^{3+}$ ions incorporated in the shell layer can harvest the excitation energy and further transfer it to $\mathrm{Yb}^{3+}$ ions in the inner core when irradiated under $808 \mathrm{~nm}$ laser. Subsequently, extracted energy of $\mathrm{Yb}^{3+}$ ions are designable to transfer to $\mathrm{Tm}^{3+}$ ions (located in ${ }^{3} \mathrm{H}_{6}$ state) in the core layer, resluting in population to ${ }^{1} \mathrm{G}_{4}$ state of $\mathrm{Tm}^{3+}$ and exhibiting blue emissions at $477 \mathrm{~nm}$ (bottom right of Fig. 4) [40,41]. According to the power dependence of room-temperature emission spectra in Fig. 6a, d, we can know that the 


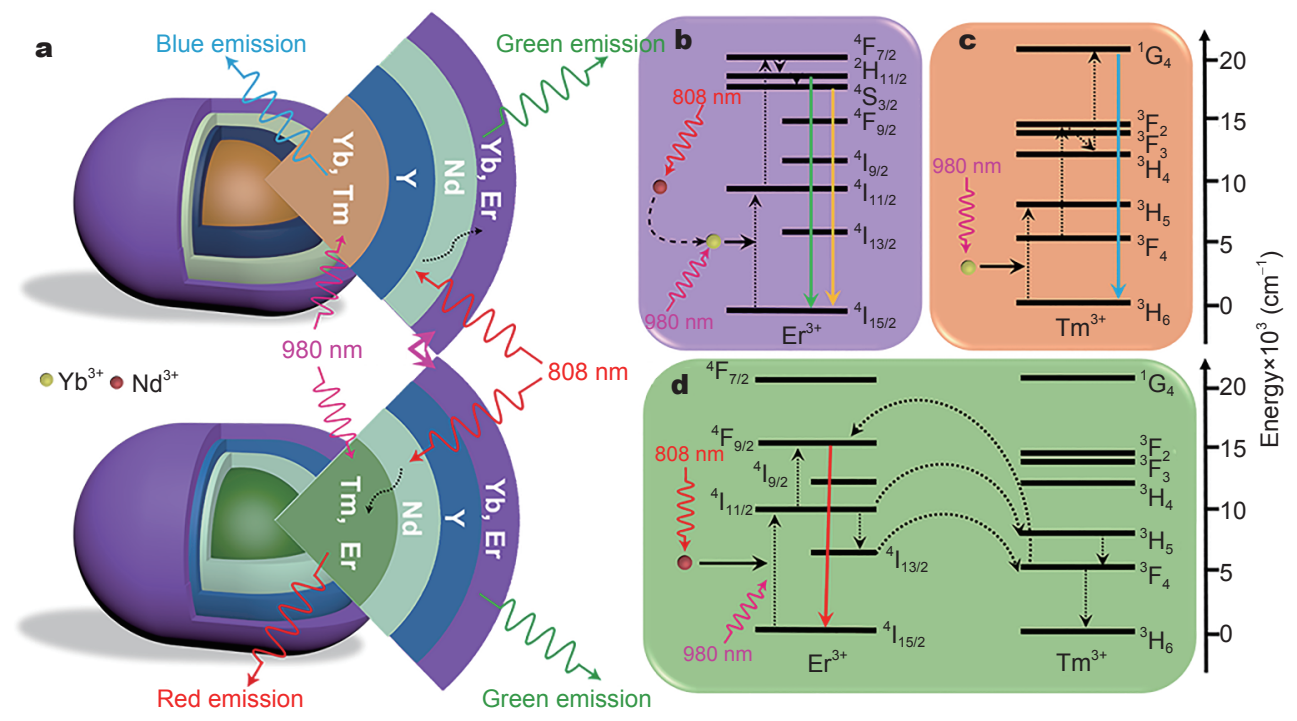

Figure 5 Schematic design detail UC process of core-triple-shell TYNE UCNCs and ETNYE UCNCs nanostructure managing complicated energy transfer.
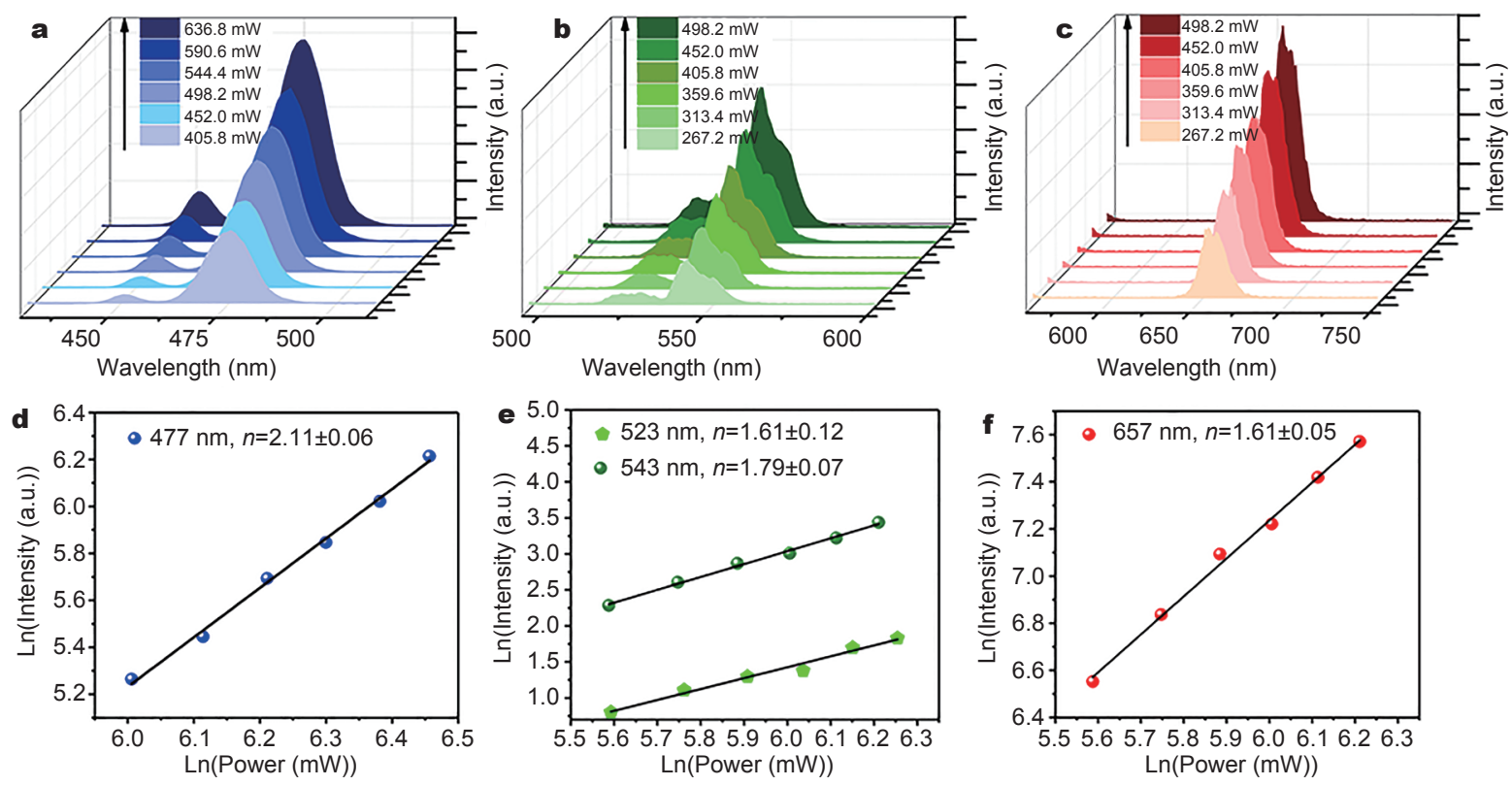

Figure 6 Power dependence of UC emissions for blue emission at $477 \mathrm{~nm}$ (a, d), green emission at 523 and $543 \mathrm{~nm}$ (b, e), and red emission at $657 \mathrm{~nm}$ (c, f) under $980 \mathrm{~nm}$ excitation.

blue emission at $477 \mathrm{~nm}$ is a UC energy transfer process involving three-photons. In response to $980 \mathrm{~nm}$ excitation, $\mathrm{Tm}^{3+}$ and $\mathrm{Er}^{3+}$ jointly act as dominating activators to absorb the transferred energy from $\mathrm{Yb}^{3+}$ ions, resulting in the excitation of blue and green emissions effectively. The green emissions located at 523 and $543 \mathrm{~nm}$ are achieved by populating the ${ }^{2} \mathrm{H}_{11 / 2}$ and ${ }^{4} \mathrm{~S}_{3 / 2}$ energy levels of $\mathrm{Er}^{3+}$ ions $[36,42,43]$. The calculated number of sequential photons $(n)$ are 1.61 and 1.79, indicating the two-step involved UC process (Fig. 6b, e), respectively. In contrast, the emissions of TYNE UCNCs centered at blue region $(477 \mathrm{~nm})$ under $980 \mathrm{~nm}$ excitation by incorporating less molar of $\mathrm{Yb}^{3+} / \mathrm{Er}^{3+}$ ions $(1 \mathrm{mmol})$ in the outermost shell. Therefore, $\mathrm{Tm}^{3+}$ ions of the inner core become prominent ions to absorb energy transmitted by $\mathrm{Yb}^{3+}$ ions and excite blue emission more effectively. As illustrated, the green 


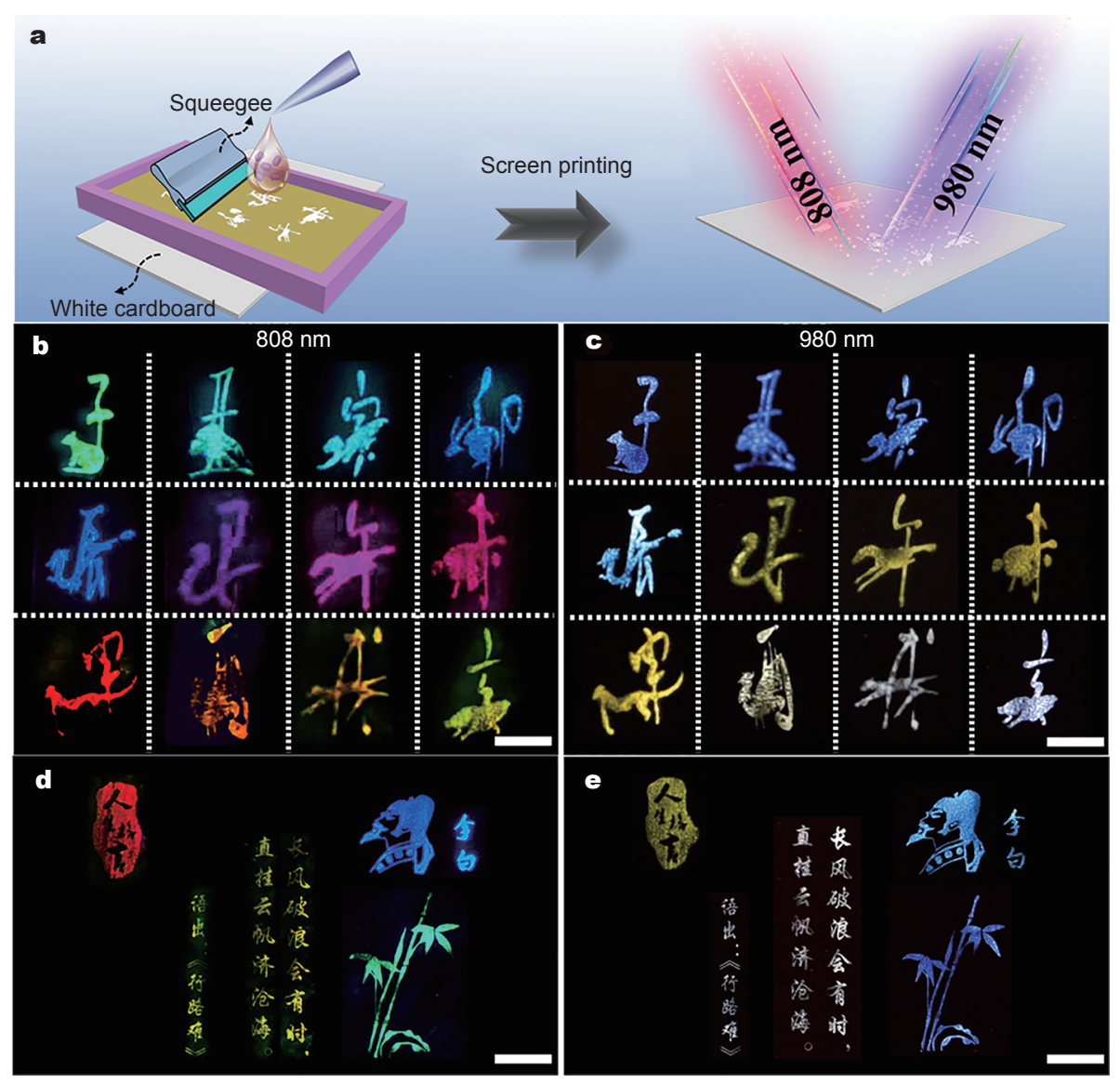

Figure 7 (a) Demonstration of screen-printed procedure of versatile and full-color display on substrate of white cardboard. Kinds of screen-printed patterns based on the physical mixing of UCNCs under (b, d) $808 \mathrm{~nm}$ and (c, f) $980 \mathrm{~nm}$ irradiation, respectively. Scale bar is $1 \mathrm{~cm}$.

color emissions can be attributed to $\mathrm{Nd}^{3+}$ sensitized procedure under $808 \mathrm{~nm}$ irradiation. The absorbed energy of $\mathrm{Nd}^{3+}$ is transferred to $\mathrm{Er}^{3+}$ through $\mathrm{Yb}^{3+}$ medium energy migration. Similarly, schematic emission engineering of ETNYE UCNCs is shown in Fig. 5. The state ${ }^{4} \mathrm{I}_{15 / 2}$ of $\mathrm{Er}^{3+}$ ions is required to absorb energy transferred from $\mathrm{Nd}^{3+}$ under $808 \mathrm{~nm}$ irradiation, and further jump to state of ${ }^{4} \mathrm{~F}_{9 / 2}$ for red emission $(657 \mathrm{~nm})$ through a cross relaxation process between $\mathrm{Tm}^{3+}$ and $\mathrm{Er}^{3+}$. Meanwhile, two emissions $\left({ }^{4} \mathrm{~S}_{3 / 2} \rightarrow{ }^{4} \mathrm{I}_{15 / 2}\right.$ at $543 \mathrm{~nm}$ and ${ }^{2} \mathrm{H}_{11 / 2} \rightarrow{ }^{4} \mathrm{I}_{15 / 2}$ at $523 \mathrm{~nm})$ collaborate with red emission $\left({ }^{4} \mathrm{~F}_{9 / 2} \rightarrow{ }^{4} \mathrm{I}_{15 / 2}\right.$ at $657 \mathrm{~nm}$, a two-photo process (Fig. 6c, f) ) of $\mathrm{Er}^{3+}$ upon excitation at $980 \mathrm{~nm}$. As aforementioned, all mechanisms are clearly ascribed to color-tunable of $\mathrm{Tm}^{3+}$ and $\mathrm{Er}^{3+}$ emission, along with the absorption of $808 \mathrm{~nm}$ photons by $\mathrm{Nd}^{3+}$ and sequential transfer to $\mathrm{Yb}^{3+}$ ions or direct absorption of $980 \mathrm{~nm}$ photons by $\mathrm{Yb}^{3+}$ ions.

Taking advantage of laser-induced multiple fluorescence characteristics of core-triple-shell UCNCs, multistage coding and full color patterns can be implemented and replace the single model of security mark. To achieve this concept, we dispersed the as-obtained UCNCs by additive mixing into PAA to formulate twelve types of UCNCs inks for further using. As a template-based printing technology, the screen printing provides us with a convenient, low-lost and efficient way for fabricating patterns. The detail screen-printing procedure of versatile and full-color patterns on substrate of white cardboard is depicted in Fig. 7. The as-obtained UCNCs inks are dripped onto the blank area primarily and subsequently continuously squeegee three times for printing designable patterns. And the entire screen plates are shown in Fig. S6a-c, where the printing patterns are magnified in Fig. S6d-e. As expected, under irradiation of $808 \mathrm{~nm}$ laser, a series of multi-color Chinese zodiac patterns are vividly presented on the substrate of white cardboards, such as green mouse, blue dragon and red monkey. Interestingly, another chain of colorful Chinese zodiac patterns are distinguished from previous patterns in response to $980 \mathrm{~nm}$ excitation, such as blue mouse, cyan dragon and 


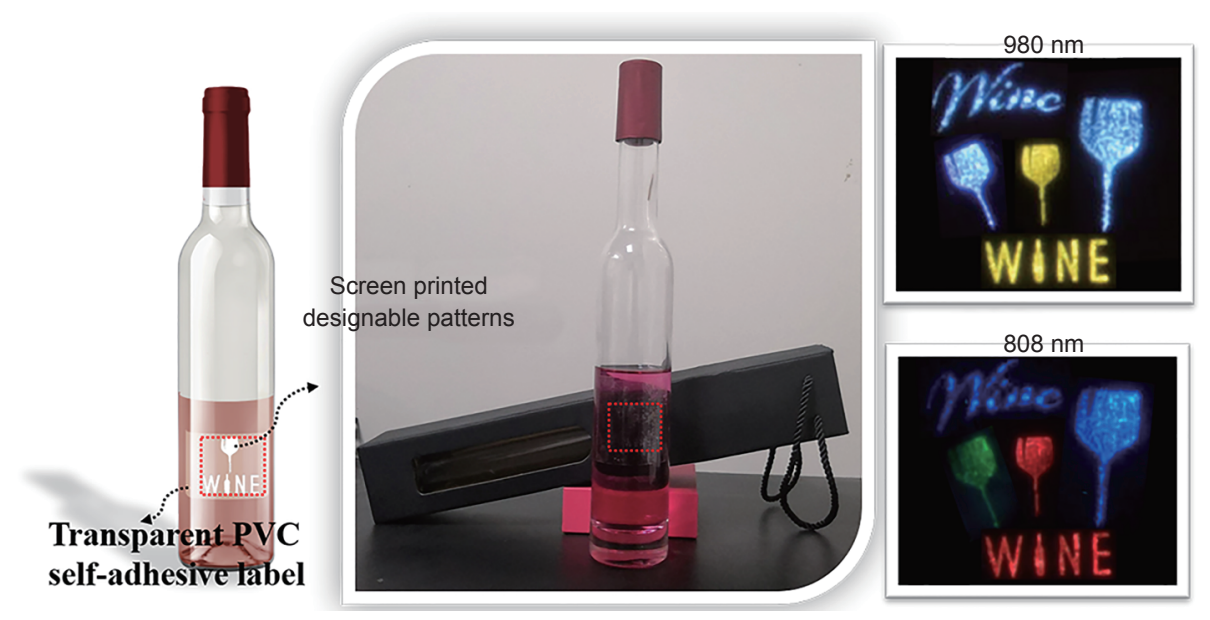

Figure 8 Screen-printed designable patterns on transparent PVC self-adhensive label for bottle of wine security application.

yellow monkey. Meaningfully, all emission colors of patterns are dependent on the mixing ratio of UCNCs, which is consistent with the UC fluorescence photography of colloidal solution (Fig. 3d). Additionally, fullcolor, large-area and high-resolution images are also generated by using the overprinting approach, just like the patterns of famous ancient poet "Li Bai" in Tang dynasty and poetry patterns of "Traveling is hard" (Fig. $7 \mathrm{~d}, \mathrm{e})$. Furthermore, as a prototype of the concept for packaging-oriented anti-counterfeiting, the as-obtained core-triple-shell UCNCs inks are screen-printed to designable and versatile patterns on transparent PVC selfadhesive label (screen plate is shown in Fig. S6c, f). The fabricated labels are attached on the bottle of wine for protecting switch of authentic information, as shown in Fig. 8. All the screen-printed patterns and complex images are transparent under visible light, while displaying multicolor and diverse emissions under 980 and $808 \mathrm{~nm}$ irradiation, respectively. Undoubtedly, printing full-color labels based on fancy fluorescence properties of coremulti-shell UCNCs should be of great significance for developing high-level security and substituting traditional single model encoding.

\section{CONCLUSIONS}

Three types of core-triple-shell TNYE, TYNE and ETNYE UCNCs with distinguishing optical properties are fabricated through modified high-temperature coprecipitation process, achieving unusual multi-color emissions for potential security printing under NIR light irradiation. The TNYE UCNCs, TYNE and ETNYE UCNCs with uniform short rod shape structure exhibit pure blue, green and red emission colors under the excitation of
$808 \mathrm{~nm}$ laser. And they also present cyan, blue and yellow emission colors when responding to $980 \mathrm{~nm}$ irradiation. Furthermore, the straightforward approach of additive mixing various volume ratio of RGB color models provides an efficient way to produce broader array of color gamut than sRGB and printing CMY. Note that blue emission is contributed to three-step luminescence of $\mathrm{Tm}^{3+}$. Two-photos UC processes of $\mathrm{Er}^{3+}$ respond to green and red emissions. The unique UPL features of core-triple-shell UCNCs endow them with applications in fullcolor security designs, verified by a series of screenprinted multi-color patterns of Chinese zodiac and largearea complex images. And transparent PVC self-adhesive anti-counterfeiting label is fabricated for the wine package, envisioning that full-color and multiple encoding patterns based on core-triple-shell UCNCs can open the door to substitute current single model encoding for security application.

Received 12 June 2018; accepted 13 August 2018; published online 4 September 2018

1 Han S, Bae HJ, Kim J, et al. Lithographically encoded polymer microtaggant using high-capacity and error-correctable QR code for anti-counterfeiting of drugs. Adv Mater, 2012, 24: 5924-5929

2 Höppe HA. Recent developments in the field of inorganic phosphors. Angew Chem Int Ed, 2009, 48: 3572-3582

3 Wu W. Inorganic nanomaterials for printed electronics: A review. Nanoscale, 2017, 9: 7342-7372

4 Smith AF, Skrabalak SE. Metal nanomaterials for optical anticounterfeit labels. J Mater Chem C, 2017, 5: 3207-3215

5 Lee J, Bisso PW, Srinivas RL, et al. Universal process-inert encoding architecture for polymer microparticles. Nat Mater, 2014, 13: 524-529

6 Kumar P, Singh S, Gupta BK. Future prospects of luminescent nanomaterial based security inks: From synthesis to anti-coun- 
terfeiting applications. Nanoscale, 2016, 8: 14297-14340

7 You M, Lin M, Wang S, et al. Three-dimensional quick response code based on inkjet printing of upconversion fluorescent nanoparticles for drug anti-counterfeiting. Nanoscale, 2016, 8: 1009610104

8 Zhou L, Wang R, Yao C, et al. Single-band upconversion nanoprobes for multiplexed simultaneous in situ molecular mapping of cancer biomarkers. Nat Commun, 2015, 6: 6938

9 Gorris HH, Wolfbeis OS. Photon-upconverting nanoparticles for optical encoding and multiplexing of cells, biomolecules, and microspheres. Angew Chem Int Ed, 2013, 52: 3584-3600

10 Meruga JM, Baride A, Cross W, et al. Red-Green-Blue printing using luminescence-upconversion inks. J Mater Chem C, 2014, 2: 2221-2227

11 Kumar P, Dwivedi J, Gupta BK. Highly luminescent dual mode rare-earth nanorod assisted multi-stage excitable security ink for anti-counterfeiting applications. J Mater Chem C, 2014, 2: 1046810475

12 Chen O, Shelby DE, Yang Y, et al. Excitation-intensity-dependent color-tunable dual emissions from manganese-doped CdS/ZnS core/shell nanocrystals. Angew Chem Int Ed, 2010, 49: 1013210135

13 Kim TH, Cho KS, Lee EK, et al. Full-colour quantum dot displays fabricated by transfer printing. Nat Photon, 2011, 5: 176-182

14 Sun YP, Zhou B, Lin Y, et al. Quantum-sized carbon dots for bright and colorful photoluminescence. J Am Chem Soc, 2006, 128: 7756-7757

15 De Cremer G, Sels BF, Hotta J, et al. Optical encoding of silver zeolite microcarriers. Adv Mater, 2010, 22: 957-960

16 Blumenthal T, Meruga J, Stanley May P, et al. Patterned directwrite and screen-printing of NIR-to-Visible upconverting inks for security applications. Nanotechnology, 2012, 23: 185305

17 You M, Zhong J, Hong Y, et al. Inkjet printing of upconversion nanoparticles for anti-counterfeit applications. Nanoscale, 2015, 7: 4423-4431

18 Tian Q, Yao W, Wu Z, et al. Full-spectrum-activated Z-scheme photocatalysts based on $\mathrm{NaYF}_{4}: \mathrm{Yb}^{3+} / \mathrm{Er}^{3+}, \mathrm{TiO}_{2}$ and $\mathrm{Ag}_{6} \mathrm{Si}_{2} \mathrm{O}_{7}$. J Mater Chem A, 2017, 5: 23566-23576

19 Liu X, Wang Y, Li X, et al. Binary temporal upconversion codes of $\mathrm{Mn}^{2+}$-activated nanoparticles for multilevel anti-counterfeiting. Nat Commun, 2017, 8: 899

20 Yao W, Tian Q, Liu J, et al. Large-scale synthesis and screen printing of upconversion hexagonal-phase $\mathrm{NaYF}_{4}: \mathrm{Yb}^{3+}, \mathrm{Tm}^{3+} / \mathrm{Er}^{3+}$ / $\mathrm{Eu}^{3+}$ plates for security applications. J Mater Chem C, 2016, 4: 6327-6335

21 Bünzli JCG, Piguet C. Taking advantage of luminescent lanthanide ions. Chem Soc Rev, 2005, 34: 1048-1077

22 Gai S, Li C, Yang P, et al. Recent progress in rare earth micro/ nanocrystals: Soft chemical synthesis, luminescent properties, and biomedical applications. Chem Rev, 2014, 114: 2343-2389

23 Mai HX, Zhang YW, Si R, et al. High-quality sodium rare-earth fluoride nanocrystals: controlled synthesis and optical properties. J Am Chem Soc, 2006, 128: 6426-6436

24 Tian Q, Yao W, Wu W, et al. Efficient UV-vis-NIR responsive upconversion and plasmonic-enhanced photocatalyst based on lanthanide-doped $\mathrm{NaYF}_{4} / \mathrm{SnO}_{2} / \mathrm{Ag}$. ACS Sustain Chem Eng, 2017, 5: 10889-10899

25 Yao W, Tian Q, Liu J, et al. Preparation and RGB upconversion optic properties of transparent anti-counterfeiting films. Nanoscale, 2017, 9: 15982-15989
26 Liu Y, Tu D, Zhu H, et al. Lanthanide-doped luminescent nanoprobes: Controlled synthesis, optical spectroscopy, and bioapplications. Chem Soc Rev, 2013, 42: 6924-6958

27 Zhou B, Tao L, Chai Y, et al. Constructing interfacial energy transfer for photon up- and down-conversion from lanthanides in a core-shell nanostructure. Angew Chem, 2016, 128: 12544-12548

28 Chen X, Peng D, Ju Q, et al. Photon upconversion in core-shell nanoparticles. Chem Soc Rev, 2015, 44: 1318-1330

29 Chan EM, Levy ES, Cohen BE. Rationally designed energy transfer in upconverting nanoparticles. Adv Mater, 2015, 27: 5753-5761

30 Li M, Yao W, Liu J, et al. Facile synthesis and screen printing of dual-mode luminescent $\mathrm{NaYF}_{4}: \mathrm{Er}, \mathrm{Yb}(\mathrm{Tm}) /$ carbon dots for anticounterfeiting applications. J Mater Chem C, 2017, 5: 6512-6520

31 Chen G, Yang C, Prasad PN. Nanophotonics and nanochemistry: Controlling the excitation dynamics for frequency up- and downconversion in lanthanide-doped nanoparticles. Acc Chem Res, 2013, 46: 1474-1486

32 Cheng ZY, Liu Y, Chang CC, et al. Authenticated rfid security mechanism based on chaotic maps. Security Comm Networks, 2013, 6: 247-256

33 Ni M, Peng H, Liao Y, et al. 3D image storage in photopolymer/ $\mathrm{ZnS}$ nanocomposites tailored by "photoinitibitor". Macromolecules, 2015, 48: 2958-2966

34 Peng H, Bi S, Ni M, et al. Monochromatic visible light "photoinitibitor": Janus-faced initiation and inhibition for storage of colored 3D images. J Am Chem Soc, 2014, 136: 8855-8858

35 Wen $\mathrm{H}$, Zhu $\mathrm{H}$, Chen $\mathrm{X}$, et al. Upconverting near-infrared light through energy management in core-shell-shell nanoparticles. Angew Chem Int Ed, 2013, 52: 13419-13423

36 Li X, Guo Z, Zhao T, et al. Filtration shell mediated power density independent orthogonal excitations-emissions upconversion luminescence. Angew Chem Int Ed, 2016, 55: 2464-2469

37 McGavin D, Stukenborg B, Witkowski M. Color figures in BJ: RGB versus CMYK. Biophys J, 2005, 88: 761-762

38 Xie X, Li Z, Zhang Y, et al. Emerging $\approx 800 \mathrm{~nm}$ excited lanthanidedoped upconversion nanoparticles. Small, 2017, 13: 1602843

39 Wang YF, Liu GY, Sun LD, et al. $\mathrm{Nd}^{3+}$-sensitized upconversion nanophosphors: efficient in vivo bioimaging probes with minimized heating effect. ACS Nano, 2013, 7: 7200-7206

40 Shen J, Chen G, Vu AM, et al. Engineering the upconversion nanoparticle excitation wavelength: cascade sensitization of tridoped upconversion colloidal nanoparticles at $800 \mathrm{~nm}$. Adv Opt Mater, 2013, 1: 644-650

41 Wang L, Li Y. Controlled synthesis and luminescence of lanthanide doped $\mathrm{NaYF}_{4}$ nanocrystals. Chem Mater, 2007, 19: 727-734

42 Li X, Wang R, Zhang F, et al. Engineering homogeneous doping in single nanoparticle to enhance upconversion efficiency. Nano Lett, 2014, 14: 3634-3639

43 Meruga JM, Cross WM, Stanley May P, et al. Security printing of covert quick response codes using upconverting nanoparticle inks. Nanotechnology, 2012, 23: 395201

Acknowledgements The work was supported by the National Natural Science Foundation of China (51471121), the Basic Research Plan Program of Shenzhen City (JCYJ20160517104459444 and JCYJ20170303170426117), the Natural Science Foundation of Jiangsu Province (BK20160383), the Fundamental Research Funds for the Central Universities (2042018kf203) and Wuhan University.

Author contributions Yao W, Tian Q and Wu W conceived the 
project and wrote the paper. Yao W performed the nanocrystal synthesis and luminescence measurements. Zheng $\mathrm{H}$ contributed to the highresolution TEM imaging and analysis. Tian $\mathrm{B}$, Li M, Wang $\mathrm{H}$, Zeng $\mathrm{P}$, and Liu L provided input into the design of the experiments and the preparation of the manuscript.
Conflict of interest The authors declare no competing financial interests.

Supplementary information Supporting data are available in the online version of the paper.

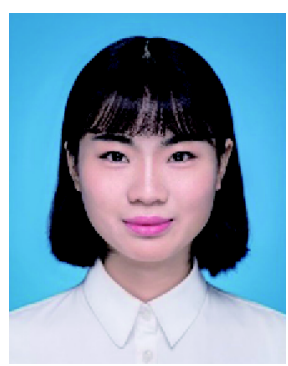

Weijing Yao received her BSc in 2015 from Inner Mongolia Agricultural University. She is currently pursuing a PhD degree under the supervision of Prof. Wei Wu in the School of Printing and Packaging at Wuhan University. Her research involves the design and synthesis of upconversion materials for anti-counterfeiting applications.
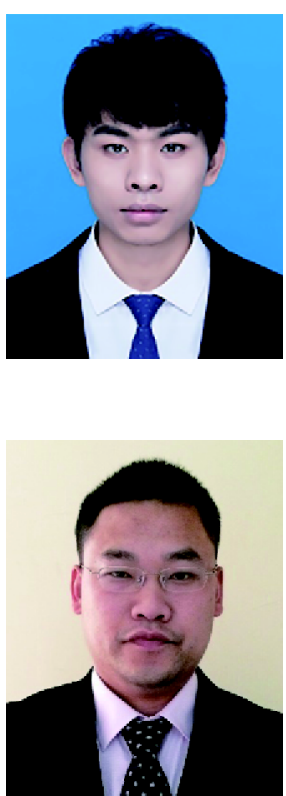

Qingyong Tian received his BSc degree in Hubei University in 2013. He is currently pursuing a $\mathrm{PhD}$ degree under the supervision of Prof. Changzhong Jiang and Prof. Wei Wu in the School of Physics and Technology at Wuhan University. His research focuses on the design and synthesis of multifunctional nanostructured materials for applications in photocatalysts and advanced energy devices.

Wei Wu received his PhD in 2011 from the Department of Physics, Wuhan University, China. He then joined the group of Prof. Daiwen Pang at Wuhan University (2011) and Prof. V. A. L. Roy at the City University of Hong Kong (2014) as a postdoctoral fellow. Now he is the full professor and Director of Laboratory of Printable Functional Nanomaterials and Printed Electronics, Vice Dean of School of Printing and Packaging, Wuhan University. He has published over 80 papers, which have received over 3000 citations. He is also the topical editor of Frontiers in Materials. His research interests include the synthesis and application of functional nanomaterials, printed electronics and intelligent packaging.

\section{用于安全编码的双模式上转换纳米光开关}

姚伟睛 ${ }^{1 \dagger}$, 田青勇 ${ }^{1 \dagger}$, 田彬 ${ }^{1}$, 李梦晓 ${ }^{1}$, 王焕军 ${ }^{1}$, 曾盼 ${ }^{1}$, 刘力 $^{1}$, 郑赫 $^{2}$, 吴伟 ${ }^{1,3,4^{*}}$

摘要 镧系离子掺杂的 $\mathrm{NaYF}_{4}$ 油墨由于其独特的上转换光致发光特性可以被应用到防伪领域. 本文首先通过高温共沉淀法合成了三种核壳结构的镧系离子掺杂的 $\mathrm{NaYF}_{4}$ 上转换纳米材料, 它们在 808 和 $980 \mathrm{~nm}$ 激光激发下均可以表现出差异性的上转换苂光性能. 其次, 通过物理 混合808 nm激发的不同颜色的悬溶液红绿蓝, 可以调节混合液的发光颜色, 实现全彩色显示. $808 \mathrm{~nm}$ 激发产生的颜色可以实现比 $\mathrm{sRGB}$ 和 印刷CMY颜色空间模型更宽的色域. 最后, 我们使用传统丝网印刷技术印刷出中国十二生肖图案和其他大面积的复杂图像, 印刷图案在 $808 \mathrm{~nm}$ 激发下可以呈现出一组多色连续的图案, 同时在 $980 \mathrm{~nm}$ 激发下也可呈现出另一组逐渐改变的多色图案, 而且, 印刷在透明聚氯乙烯 $\mathrm{PVC}$ 标签的图案可以附在酒瓶包装上, 实现酒瓶包装的防伪. 基于 $\mathrm{NaYF}_{4}$ 的双模式苂光图案可以替代传统单模式苂光图案, 实现高水平的 全色防伪及安全编码. 